Revista de Matemática: Teoría y Aplicaciones 2007 14(1) : 7-20

CIMPA - UCR - CCSS ISSN: 1409-2433

\title{
SELECCIÓN DE PERSONAL MEDIANTE REDES NEURONALES ARTIFICIALES
}

\author{
German Leonardo Acevedo Orduña* \\ Eduardo Francisco Caicedo Bravo ${ }^{\dagger}$ Humberto Loaiza Correa H $^{\ddagger}$
}

Recibido/Received: 20 May 2004 - Aceptado/Accepted: 19 May 2006

\begin{abstract}
Resumen
Se la optimización de los sistemas de selección de personal mediante la aplicación de técnicas de inteligencia computacional para la clasificación de patrones sicológicos. El sistema está basado en la prueba sicológica 16-PF, de donde se extraen los factores de Primer Orden y la distorsión motivacional, para ser utilizados como entradas a un algoritmo de reconocimiento de patrones que actúa como predictor de comportamiento. Inicialmente se definen la prueba 16-PF y las estructuras computacionales utilizadas. Luego se describen los procedimientos de normalización y extracción de patrones y finalmente, se proveen resultados experimentales para ilustrar el desempeo de las técnicas de clasificación, las cuales son analizadas en un problema de selección de cadetes para la Escuela Naval Almirante Padilla.
\end{abstract}

Palabras clave: Inteligencia artificial, redes neuronales, reconocimiento de patrones, selección de personal.

\begin{abstract}
We study the personnel selection problem by the application of techniques of computing intelligence for the classification of psychological patterns. The system is based on the psychological test 16-PF, for the extraction of the First Order factors and the motivational distortion, for being used as entries in a pattern recognition algorithm that intends to predict behavior. Initially, we define the $16-\mathrm{PF}$ test and the computational structures to be used. Then we describe the normalization and pattern extraction procedures, and finally, we provide experimental results for illustrating the performance of the classification techniques, which are analyzed in a problem of cadets selection for the Navy School Almirante Padilla.
\end{abstract}

\footnotetext{
*Escuela Naval de Cadetes Almirante Padilla, Cartagena, Colombia. E-Mail: acevedo@enap.edu.co

${ }^{\dagger}$ Grupo de Percepción y Sistemas Inteligentes, Escuela de Ingeniería Eléctrica y Electrónica, Universidad del Valle - A.A. 25360 Cali, Colombia. E-Mail: ecaicedo@eiee.univalle.edu.co

${ }^{\ddagger}$ Misma dirección de E. Caicedo. E-Mail: hloaiza@eiee.univalle.edu.co
} 
Keywords: Artificial intelligence, neural nets, pattern recognition, personnel selection.

Mathematics Subject Classification: $82 \mathrm{C} 32$.

\section{Introducción}

Los sistemas artificiales bioinspirados, tratan de reproducir el proceso de solución de problemas e interpretación de datos del cerebro. Es así como los humanos aplican el conocimiento previo junto con la experiencia, a la solución de nuevos problemas o situaciones. Una red neuronal artificial (RNA), toma como ejemplos los problemas resueltos para construir un sistema que permite, a través de un aprendizaje previo, tomar decisiones y realizar clasificaciones de patrones. Dados los adelantos tecnológicos en microelectrónica, los procesadores modernos pueden realizar una gran cantidad de operaciones numéricas en una corta unidad de tiempo, lo cual nos permite de cierta forma, emular el comportamiento del cerebro humano pero con la gran limitación de que no pueden ser lo suficientemente paralelos y robustos.

Por los motivos anteriormente expuestos y con base en la necesidad institucional de optimizar el uso del recurso humano, se plantea la búsqueda de una solución tecnológica la cual, permita disminuir los niveles de deserción del personal seleccionado para adelantar cursos de formación, como los son los Oficiales y Suboficiales de la Armada de la República de Colombia. Dentro de los principales objetivos del proyecto se destacan los siguientes:

- Experimentar bajo una visión futurista y real con la aplicación de Sistemas Inteligentes, para optimizar los procesos de administración de personal.

- Disminuir los niveles de subjetividad en la selección del personal.

- Hacer un estudio detallado del comportamiento estadístico de deserción de los cadetes, con respecto al resultado de las evaluaciones sicológicas de ingreso.

\section{Marco teórico}

\subsection{Redes neuronales artificiales}

La teoría y modelado de redes neuronales artificiales, está inspirada en la estructura y funcionamiento de los sistemas nerviosos en los cuales, la neurona es el elemento fundamental. Existen neuronas de diferentes formas, tamaños y longitudes. Estos atributos son importantes para determinar la función y utilidad de la neurona. La clasificación de estas células en tipos estándar, ha sido realizada por muchos neuroanatomistas. Las redes neuronales artificiales, son redes interconectadas masivamente en paralelo, formadas por elementos simples las cuales, intentan interactuar con los objetos del mundo real del mismo modo que lo hace el sistema nervioso biológico.

Las redes neuronales son modelos que intentan reproducir el comportamiento del cerebro. Como tal, el modelo realiza una simplificación, averiguando cuáles son los elementos 
relevantes del sistema, bien porque la cantidad de información de que se dispone es excesiva o bien porque es redundante. Una elección adecuada de sus características, más una estructura conveniente, es el procedimiento convencional utilizado para construir redes capaces de realizar una determinada tarea [1]. Cualquier modelo de red neuronal, consta de dispositivos elementales de proceso: las neuronas. A partir de ellas, se pueden generar representaciones específicas, de tal forma que un estado conjunto de ellas, puede significar una letra, un número o cualquier objeto. Generalmente, se pueden encontrar tres tipos de neuronas de acuerdo con su función:

1. Neuronas de entrada: Son aquellas que reciben estímulos relacionados con el aparato sensorial y que tomarán la información de entrada a la red.

2. Neuronas ocultas: La información se transmite a ciertos elementos internos que se ocupan de su procesado. Es en las sinapsis y las neuronas correspondientes a este segundo nivel, donde se genera cualquier tipo de representación interna de la información. Estos elementos se denominan unidades ocultas, puesto que no tienen relación directa con la información de entrada ni con la de salida.

3. Neuronas de salida: Una vez finalizado el período de procesado, la información llega a las unidades de salida, cuya misión es entregar la respuesta del sistema, al sistema actuante.

En la Figura 1 se presenta la arquitectura típica de una red neuronal tipo perceptrón multicapa. Se conoce como capa, un nivel o un conjunto de neuronas cuyas entradas provienen de la misma fuente (que puede ser otra capa de neuronas) y cuyas salidas se dirigen al mismo destinn (nue nuede ser ntra cana de newronas)

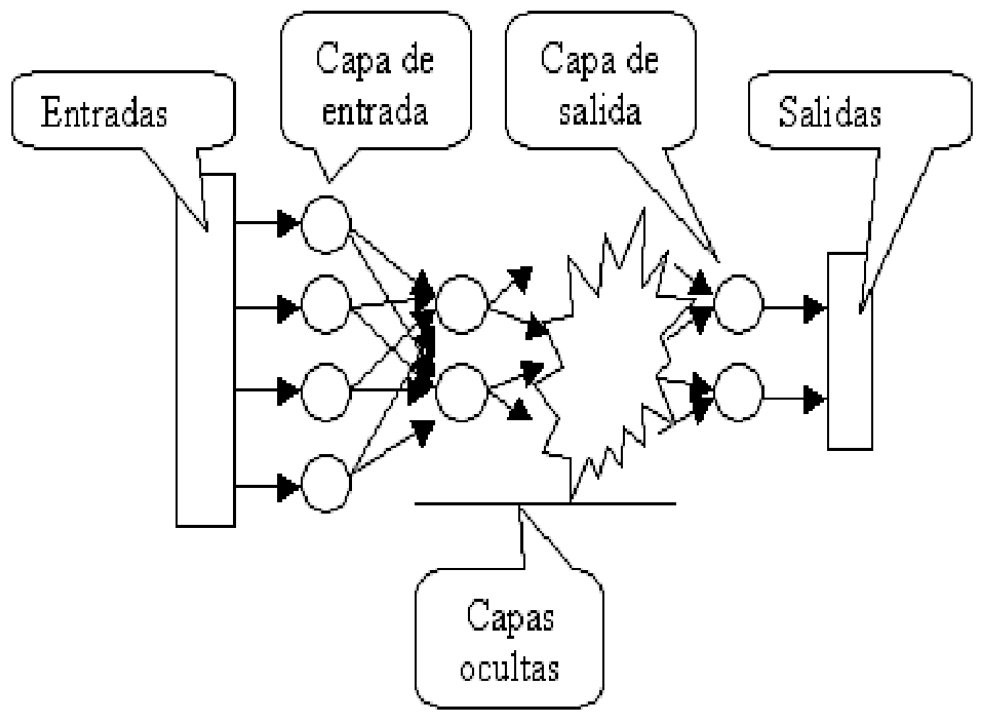

Figura 1: Red Neuronal tipo Perceptrón Multicapa (MLN-NN)

Todas las neuronas que componen la red se hallan en cierto estado. En una visión simplificada, podemos decir que hay dos posibles estados, reposo y excitado, a los que 
denominaremos globalmente estados de activación y a cada uno de los cuales, se le asigna un valor. Los valores de activación pueden ser continuos o discretos, además, estos pueden ser limitados o ilimitados. Si son discretos, suelen tomar un conjunto pequeño de valores o bien, valores binarios. En notación binaria, un estado activo se indicaría por un 1 y se caracteriza por la emisión de un impulso por parte de la neurona (potencial de acción), mientras que un estado pasivo se indicaría por un 0 y significaría que la neurona está en reposo. En otros modelos se considera un conjunto continuo de estados de activación, en lugar de sólo dos Estados, en cuyo caso se les asigna un valor entre $[0,1]$ o en el intervalo $[-1,1]$, generalmente siguiendo una función matemática.

Existen muchas definiciones del concepto general de aprendizaje, una de ellas podría ser: la modificación del comportamiento inducido por la interacción con el entorno y como resultado de experiencias conducente al establecimiento de nuevos modelos de respuesta a estímulos externos. Esta definición fue enunciada muchos aos antes de que surgieran las redes neuronales artificiales, sin embargo, puede ser aplicada también a los procesos de aprendizaje de estos sistemas. Biológicamente, se suele aceptar que la información memorizada en el cerebro está más relacionada con los valores sinápticos de las conexiones entre las neuronas que con ellas mismas, es decir, el conocimiento se encuentra en las sinapsis. En el caso de las redes neuronales artificiales, se puede considerar que el conocimiento se encuentra representado en los pesos de las conexiones entre neuronas. Todo proceso de aprendizaje implica cierto número de cambios en estas conexiones. En realidad, puede decirse que se aprende modificando los valores de los pesos de la red.

$\mathrm{Al}$ igual que el funcionamiento de una red depende del número de neuronas de las que disponga y de cómo estén conectadas entre sí, cada modelo dispone de sus propias técnicas de aprendizaje.

Existen cuatro aspectos que caracterizan una red neuronal: su topología, el mecanismo de aprendizaje, el tipo de asociación que realiza entre la información de entrada y salida y por último, la forma de representación de estas informaciones.

De acuerdo a la topología de las redes neuronales, estas se pueden clasificar como redes monocapa o redes multicapa. Con referencia al mecanismo de aprendizaje, se pueden distinguir las redes neuronales con aprendizaje supervisado y las redes neuronales con aprendizaje no supervisado. En cuanto al tipo de asociación entre las informaciones de entrada y salida, encontramos a las hetero-asociativas y las redes auto asociativas. En cuanto a la forma en que se representan las informaciones de entrada y las respuestas o datos de salida, encontramos las redes continuas que se caracterizan por sus entradas y salidas analógicas, las redes híbridas por sus entradas analógicas y sus salidas binarias y las redes discretas por las entradas y salidas binarias.

Las redes neuronales de base radial, son una poderosa clase de redes neuronales unidireccionales para aproximación funcional, las cuales difieren enormemente del MLP en su función de activación y la forma como son usadas. Pueden considerarse de tipo híbrido por incorporar aprendizaje supervisado y no supervisado. La arquitectura de una red RBF (ver figura 2) contiene una capa de neuronas de entrada (una neurona para cada componente característico), tal como el MLP, una capa oculta de neuronas, que operan con base en la distancia que separa el vector de entradas respecto al vector sináptico que cada una almacena (denominado centroide), cantidad a la que aplican una función radial con forma 
Gaussiana. En la RBF, las neuronas de la capa oculta son de respuesta localizada, pues sólo responden con una intensidad apreciable cuando el vector de entradas presentado y el centroide de la neurona, pertenecen a una zona próxima en el espacio de las entradas. Y por último se encuentra una capa de neuronas de salida con función de activación lineal, las cuales esencialmente calculan la suma ponderada de las salidas que proporciona la capa oculta.

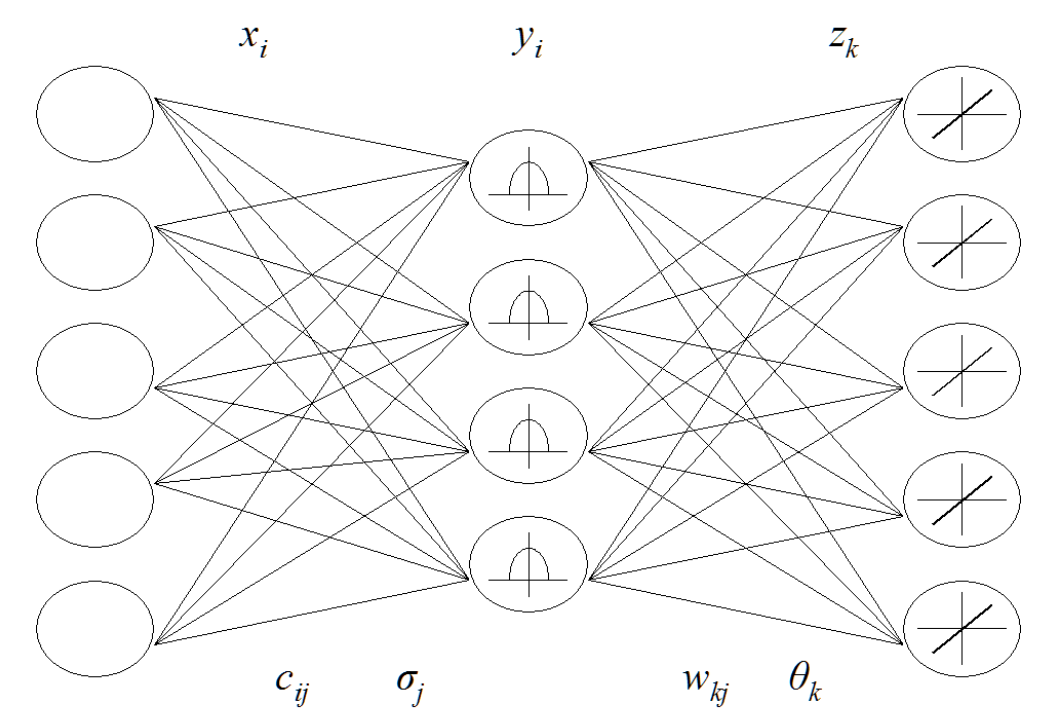

Figura 2: Red Neuronal Radial Basis Function (RBF-NN)

A continuación se describe matemáticamente el modelo de la RBF:

$$
r_{j}^{2}=\left\|x-c_{j}\right\|^{2}=\sum_{i}\left(x_{i}-c_{i j}\right)^{2}
$$

donde: $x_{i}$ son las entradas a la red, $y_{i}$ son las salidas de la capa oculta (ver figura 2), $z_{k}$ son las salidas globales de la red (ver figura 2), $c_{j i}$ es el centroide neuronas de la capa oculta y $r_{j}$ es la distancia euclídea entre las entradas y su centroide.

Cada RBF depende de las posiciones de los centros, donde este toma el mayor valor cuando es activado por una entrada $x_{i}$ cercana a $c_{j i}$. El valor de la respuesta disminuye en la medida que $x_{i}$ se aleja de $c_{j i}$. Cada RBF responde a una pequeña región del espacio de características (campo receptivo). La salida de la neurona $y_{j}$ se calcula a partir de una función de activación denominada función radial $\phi(r)$. Una de las mas típicas es la función Gaussiana:

$$
\phi(r)=e^{-r^{2} / 2 \sigma^{2}}
$$

Existen diferentes paradigmas para determinar cómo hacer el entrenamiento de las redes RBF [?]. En el caso más simple, los pesos $W$ de entrada y de salida de las neuronas ocultas, permanecen fijos, es decir que no requieren de entrenamiento. En el segundo y 
más flexible modelo, se entrenan únicamente los pesos de las neuronas de salida. El tercer modelo requiere el entrenamiento de todos los pesos, centros y otros parámetros.

\subsection{Cuestionario de personalidad de 16 Factores (16-PF)}

El 16 PF [5] es un cuestionario diseado para medir dimensiones normales de la personalidad. En el caso de los adultos, el instrumento ofrece 16 puntuaciones básicas. Dentro de la misma serie, existen disponibles otros cuestionarios que miden la personalidad de sujetos más jóvenes, incluso nios. El cuestionario de Personalidad de 16 Factores (denominado en adelante con la sigla $16 \mathrm{PF}$ ), es un instrumento de valoración objetiva, elaborado mediante investigación sicológica, con el fin de ofrecer, en el menor tiempo posible, una visión muy completa de la personalidad. El $16 \mathrm{PF}$ se diseó para ser aplicado a sujetos de 16 aos en adelante y existen, en uso o en preparación, seis formas diferentes: la $\mathrm{C}$ y la D están destinadas a sujetos con formación medio-baja; la A y la B para personas con nivel de formación igual o superior al de Bachillerato Superior. Las restantes formas del cuestionario, E y F, están proyectadas para sujetos que presentan algún déficit en su formación y nivel de comprensión lectoral. La corrección y puntualización puede realizarse manualmente o con máquinas. Existen 6 formas distintas de la prueba (ver tabla 1):

\begin{tabular}{l|clc}
\hline \hline Formas & N Items & Aplicación & Tiempo \\
\hline A/B & 187 & $\begin{array}{l}\text { Sujetos con formación equivalente a } \\
\text { educación secundaria }\end{array}$ & $45-60$ min. \\
C/D & 105 & $\begin{array}{l}\text { Sujetos con formación ligeramente } \\
\text { inferior al exigido para las formas A/B }\end{array}$ & $30-40$ min. \\
E/F & 128 & $\begin{array}{l}\text { Sujetos con bajo nivel de vocabulario } \\
\text { y nivel de formación por debajo de } \\
\text { lo normal }\end{array}$ & $30-40$ min. \\
\hline \hline
\end{tabular}

Tabla 1: Forma de aplicación para la prueba 16-PF

La versión global de la personalidad que intenta el 16 PF, se basa en la evaluación de 16 dimensiones, funcionalmente independientes y sicológicamente significativas, definidas repetidamente durante más de treinta aos de investigación factoriales, en grupos de sujetos normales clínicos. También incluye una escala DM (Distorsión Motivacional) para detectar los intentos de presentar buena imagen. Los 16 factores son los siguientes:

Factores Q : Estas escalas son menos firmes, según Cattell. No obstante, aportan valiosa información.

Q1: Rebeldía : (Conservador vs. Radicalismo). En una visión superficial puede parecer que este es un factor que presenta un gran parecido con el "E" y sin embargo, en esta escala se pone menos énfasis en la dominancia y en la hostilidad para trasladar el acento más hacia el aspecto constructivo. Es importante observar que Q1 no es meramente una rebeldía propia del adolescente, sino que expresa en sí misma formas intelectualizadas de hostilidad, comúnmente aceptada por la sociedad. 
Q2: Autosuficiencia : (Adhesión al grupo vs. Autosuficiencia). Presenta cierta relación con el factor introversión - extroversión, factor de Segundo Orden. Hemos de tener en cuenta que la dependencia del grupo no es lo mismo que la conformidad al grupo $(\mathrm{G})$.

Q3: Autocontrol : (Incontrolable vs. Autocontrol). Esta escala presenta cierta similaridad con "C" (Fuerza del Yo) y con "G" (Conformidad al Grupo), pero no aprecia la desorganización del yo que puede aparecer con C- ni tampoco presenta las implicaciones morales que pueden apreciarse con G. Los elementos propuestos en este factor intentan evaluar algo muy semejante al autocontrol de manera muy general.

Q4: Tensión : (Poca tensión vs. Mucha tensión). Es un factor con gran carga de ansiedad (flotante) muy típica de la clásica Neurosis de Ansiedad. La disposición de los diferentes elementos componentes del factor Q4, sugiere que esta tensión puede ser variable según sea percibida la situación por el sujeto que contesta el Cuestionario.

Los siguientes son los factores de Segundo Orden, los cuales son deducidos mediante ecuaciones derivadas de los factores de primer orden y que por tanto, no se tomaron en cuenta para ser introducidos como entradas a la red neuronal.

QI: Ansiedad : (Integración dinámica vs Ansiedad). Es quizá de los factores de Segundo Orden más importantes, en el que intervienen elementos correspondientes a los factores Q4+ (Tensión), O+ (Tendencia a la Culpabilidad), H- (Atrevimiento), C- (Fuerza del Yo), L+ (Suspicacia) y Q3- (Autocontrol).

QII: Extraversión : (Introversión vs. Extroversión). Refiriéndonos a la dimensión, este factor está correlacionado positivamente con puntuaciones altas en los factores A (Afectividad), F (Impulsividad) y H (Atrevimiento) y baja en Q2 (Autosuficiencia).

QIII: Socialización controlada : (Sociopatía vs. Socialización controlada). Es una versión ampliada y completada del factor G (Superego) de Primer Orden con la aportación de los factores $\mathrm{N}+$ (Astucia), Q3+ (Control) y F- (Impulsividad) y E(Dominancia).

QIV: Independencia : (Dependencia vs. Independencia). Análisis factoriales de los factores E+ (Dominancia), L+ (Suspicacia), M+ (Imaginación autística), Q1 (Rebeldía) y Q2 (Autosuficiencia).

QV: Subjetividad : (Objetividad vs. Subjetividad) entre las Escalas implicadas en este factor de Segundo Orden, se encuentra I+ (Sensibilidad emocional), M+ (Imaginación autística), E- (Dominancia), G- (Conformidad al Grupo), L+ (Suspicacia) y Q2 (Autosuficiencia).

Escalas complementarias de validez:

Escala de Distorsión Motivacional (DM) : Detecta a los sujetos que intentan ofrecer una buena imagen de sí mismos. 
Escala de Negación (o del azar) : Permite discriminar a los sujetos negativistas o poco cooperativos que contestan al azar.

\section{Experimentación y resultados}

\subsection{Diseño del experimento}

Fundamentados en la experiencia del personal de sicólogos de la Armada Nacional y los resultados de las pruebas presentadas por el personal que ha ingresado a la institución, se diseñó un experimento, el cual permite de una forma ágil y tomando como base un aprendizaje previo, reconocer los patrones característicos del personal, que durante su permanencia en la institución, ha evidenciado alta calidad en el desempeño de sus funciones, para aplicar estos criterios a la selección del personal, disminuyendo así los altos niveles de subjetividad en la selección.

El sistema de selección de personal, se desarrolló tomando como plataforma el programa Matlab. Para la experimentación, se elaboró una base de datos a partir de una muestra de población de 552 excadetes. La base de datos está conformada por los resultados de las pruebas sicológicas obtenidas al ingreso a la Escuela Naval y la evaluación del desempeño de estos durante su permanencia en la Escuela. El lapso de la muestra está comprendido por cadetes ingresados entre los años 1995 y 1998. Se tomaron como datos de entrada al sistema, los 16 factores de primer orden y la distorsión motivacional que arroja la prueba 16-PF y como salidas binarias ( $\mathrm{Sí}=1$; No $=0$ ), los correspondientes resultados de desempeño durante la permanencia en la Escuela Naval de la muestra así:

- El individuo obtuvo el grado de Teniente de Corbeta. (Sí = 1; No =0)

- Fue retirado por Falta de aptitudes para la carrera. (Sí = 1; No =0)

- Fue retirado por solicitud propia. (Sí = $1 ;$ No $=0$ )

- Fue retirado por pérdida de curso. (Sí = 1; No =0)

- Fue retirado por incapacidad física. $(\mathrm{Sí}=1 ; \mathrm{No}=0)$

La información anterior se normalizó para valores entre 0 y 1 para facilitar la convergencia de la red, mediante el siguiente método:

$$
\mathbf{x}_{i}=\frac{x(n)-\mu}{\sigma}
$$

donde: $\mathbf{x}_{i}$ es el valor normalizado, $\mu$ es el valor promedio y $\sigma$ es la desviación estándar.

Debido a resultados de experimentación, no fueron tenidos en cuenta los factores de segundo orden que arroja la prueba, por ser estos resultados de operaciones matemáticas entre factores de primer orden. Dadas las capacidades de generalización de la red neuronal, se deduce que la inclusión de los factores de segundo orden como variables de entrada, se constituye como una redundancia en la información. 


\subsection{Análisis de datos}

Se procedió a hacer una graficación de las curvas de los perfiles de los grupos de clasificación a saber:

- Cadetes graduados (302 patrones)

- Cadetes retirados por falta de aptitudes para la carrera (35 patrones)

- Cadetes retirados por solicitud propia (153 patrones)

- Cadetes retirados por pérdida de curso (44 patrones)

- Cadetes retirados por incapacidad física (18 patrones)

Luego se obtuvo la media de cada uno de los decatipos, permitiendo obtener el promedio de los perfiles determinados por los dieciseis factores primarios que arroja la prueba.

Dado que se encontró un alto índice de distorsión motivacional a la prueba, se decidió segmentar la muestra para trabajar el modelo sólo con individuos que presentarán pruebas de personal con un buen índice de confiabilidad.

La muestra seleccionada para el trabajo, fue la de los individuos que presentaban valores de distorsión motivacional de 10 o inferior (ver tabla 2).

\begin{tabular}{l|ccccccc}
\hline \hline & \multicolumn{7}{|c}{$\%$ Distorsión Motivacional } \\
\hline & 4 & 6 & 8 & 10 & 12 & 14 & 15 \\
Graduados & 9 & 30 & 69 & 116 & 190 & 273 & 302 \\
Falta de aptitudes & 2 & 2 & 5 & 14 & 23 & 31 & 35 \\
Solicitud propia & 15 & 27 & 49 & 86 & 112 & 143 & 153 \\
Pérdida de curso & 4 & 8 & 13 & 25 & 32 & 38 & 44 \\
Incapacidad física & 0 & 2 & 4 & 9 & 16 & 18 & 18 \\
Porcentajes & 5,4 & 12,5 & 25,3 & 45,2 & 67,5 & 91,1 & 100,0 \\
\hline \hline
\end{tabular}

Tabla 2: Segmentación de la muestra por Distorsión Motivacional

Como parte del análisis de la muestra, se consideró la comparación de las medias matemáticas (ver figura 3) y las desviaciones estándar (ver figura 4) de los valores de los factores primarios, con el fin de identificar las variables o factores que tienen una mayor incidencia en el modelo.

\subsection{Arquitectura y entrenamiento de los clasificadores}

Para realizar la clasificación de los patrones sicológicos, se seleccionaron dos tipos diferentes de redes neuronales artificiales, ambas de entrenamiento supervisado y estructura multicapa. Dichas arquitecturas son el Perceptrón Multicapa (Multilayer Perceptrón MLP-NN), y las redes neuronales de Base Radial (Radial Basis Function RBF-NN). 


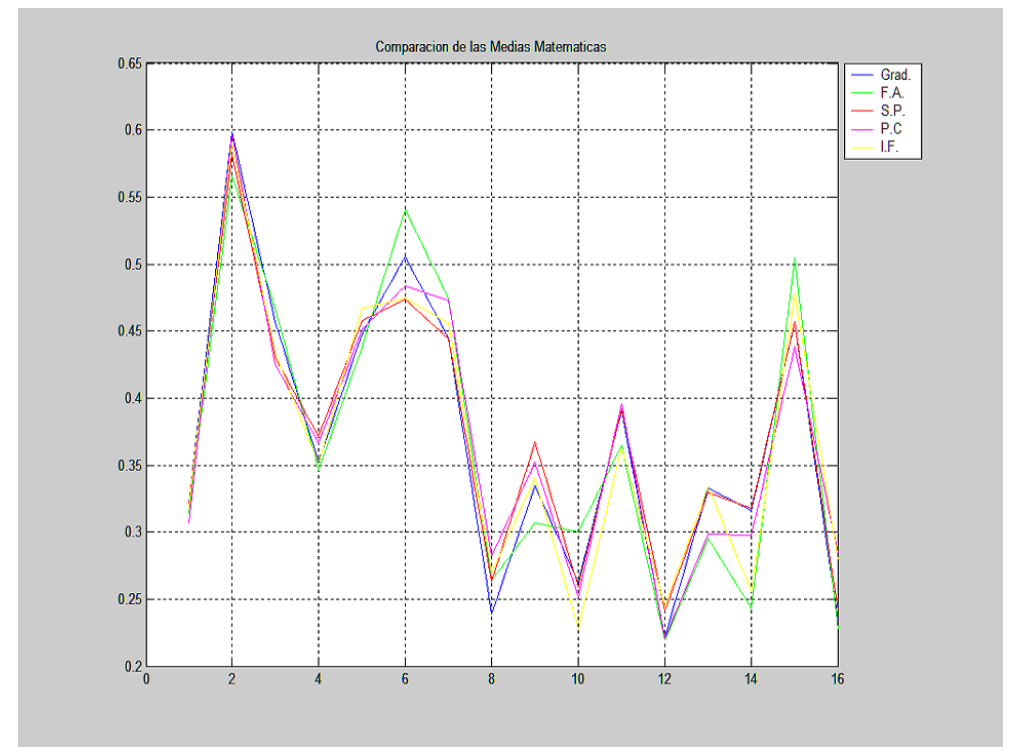

Figura 3: Comparación de las medias matemáticas

\subsubsection{Redes Neuronales tipo Perceptrón Multicapa}

Para la implementación del sistema basado en redes neuronales tipo Perceptrón Multicapa (MLP), se utilizó la siguiente arquitectura determinada mediante experimentación:

1. Capa de entrada: 17 neuronas que reciben la información de los 16 factores primarios y la distorsión motivacional.

2. Capa oculta: 18 neuronas con función de activación logarítmica-hiperbólica.

3. Capa de salida: 1 neurona con función de activación lineal.

La red fue entrenada durante 100 iteraciones mediante el algoritmo de LevenbergMarquard, con un total de 200 perfiles sicológicos correspondientes a cadetes ingresados desde 1995 hasta 1998 (ver figura 5).

\subsubsection{Redes Neuronales de Base Radial}

Para la implementación del sistema basado en redes neuronales de base radial, se utilizó la siguiente arquitectura determinada mediante experimentación:

1. Capa de entrada: 17 neuronas que reciben la información de los 16 factores primarios y la distorsión motivacional.

2. Capa oculta: 175 neuronas con función de activación Gaussiana con una desviación estándar de 0,85 determinada mediante experimentación. 


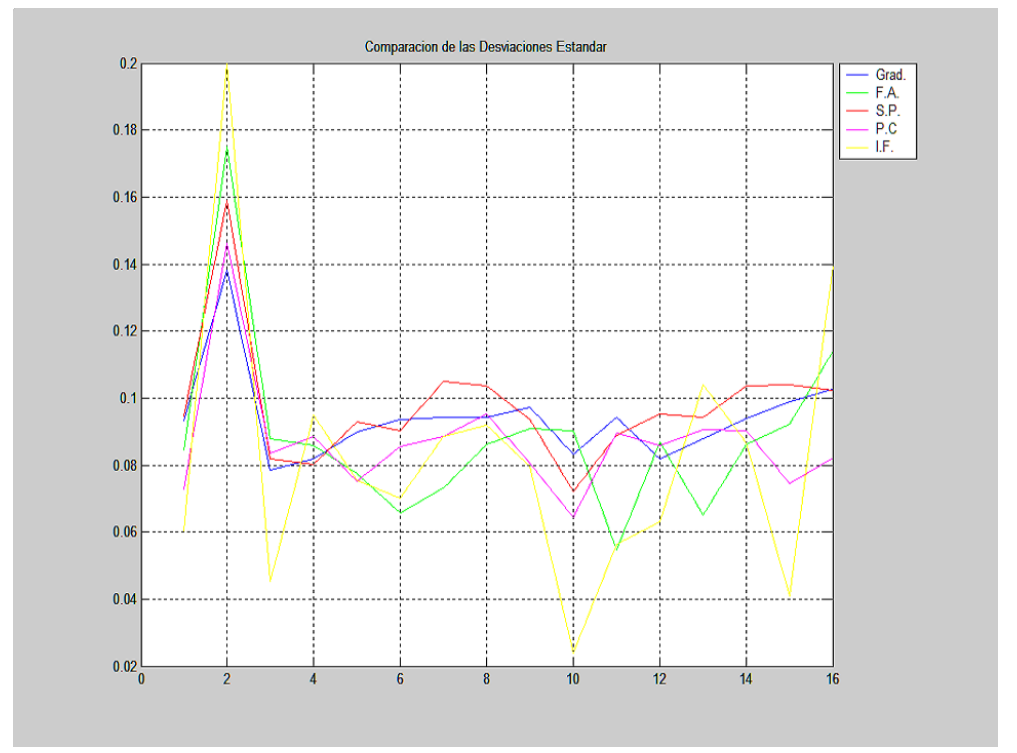

Figura 4: Comparación de las desviaciones estándar

3. Capa de salida: 1 neuronas con función de activación lineal.

La red fue entrenada con un total de 200 perfiles sicológicos correspondientes a cadetes ingresados desde 1995 hasta 1998 (ver figura 6).

\subsection{Resultados de validación}

Para validar las redes se usaron 50 perfiles sicológicos que no fueron usados durante el entrenamiento, correspondientes a cadetes ingresados en 1998, con el fin de verificar la capacidad de la red neuronal, para predecir el comportamiento de nuevos individuos, basándose en la generalización de los patrones presentados para el entrenamiento. Se determinó mediante experimentación el valor de 0.6 como nivel de umbral de detección.

\begin{tabular}{l|c}
\hline \hline Sistema de Clasificación & Mayor Eficiencia \\
MLP-NN & $66 \%$ \\
RBF-NN & $70 \%$ \\
Empírico & $54,7 \%$ \\
\hline \hline
\end{tabular}

Tabla 3: Validación de redes neuronales

El error de validación del sistema, es obtenido mediante la siguiente ecuación:

$$
\text { Error }=\sum_{i=1}^{N} \sum_{j=1}^{4}\left(Y d_{i}-Y r_{j}\right)^{2}
$$




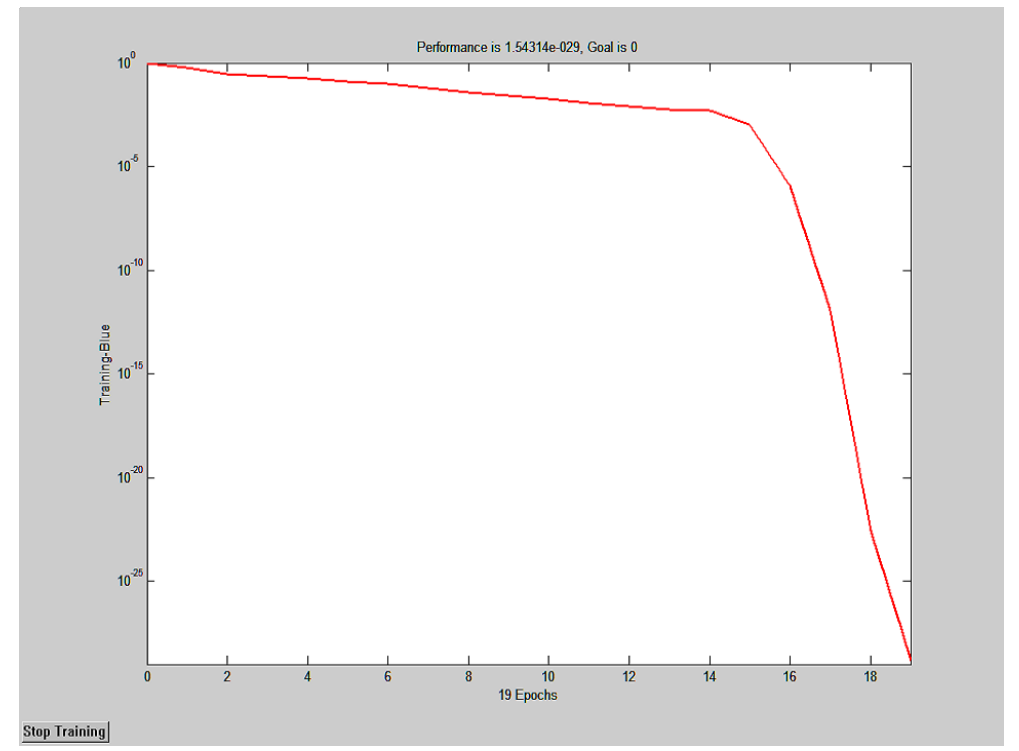

Figura 5: Entrenamiento de la red MLP

donde: $Y d_{i}$ es la salida deseada, $Y r_{j}$ es la salida de la Red Neuronal, $N$ es el número de individuos y $j$ es el número de salidas.

El mínimo error global obtenido durante la validación es del 30\%, es decir, a pesar de la escasa basa de datos con la que se cuenta hasta el momento, el sistema es capaz de predecir el desempeño de un aspirante a ingresar a la Escuela Naval con una posibilidad de acierto de aproximadamente $70 \%$.

\subsection{Proyección del proyecto}

El sistema permitirá en el futuro, agilizar y sistematizar el trabajo de las comisiones de selección de personal para la Armada Nacional en todos los niveles. En la medida que el sistema sea alimentado con un mayor número de patrones, se podrá disminuir el umbral de error y subjetividad en la selección del personal.

Con base en los resultados obtenidos, se propone el uso de algoritmos híbridos de clasificación que permitan aumentar el índice de eficiencia del sistema. Así mismo, se requiere involucrar variables adicionales que permitan mejorar la eficiencia del modelo. Tales variables pueden ser de orden académico, social o físico.

\section{Conclusiones}

El desarrollo de un sistema de inteligencia computacional, permite agilizar los procesos de selección para el personal que desea ingresar a la Armada Nacional, permitiendo con base en la extracción de las características sicológicas de una población conocida, hacer una predicción de la permanencia del nuevo individuo en la institución. 


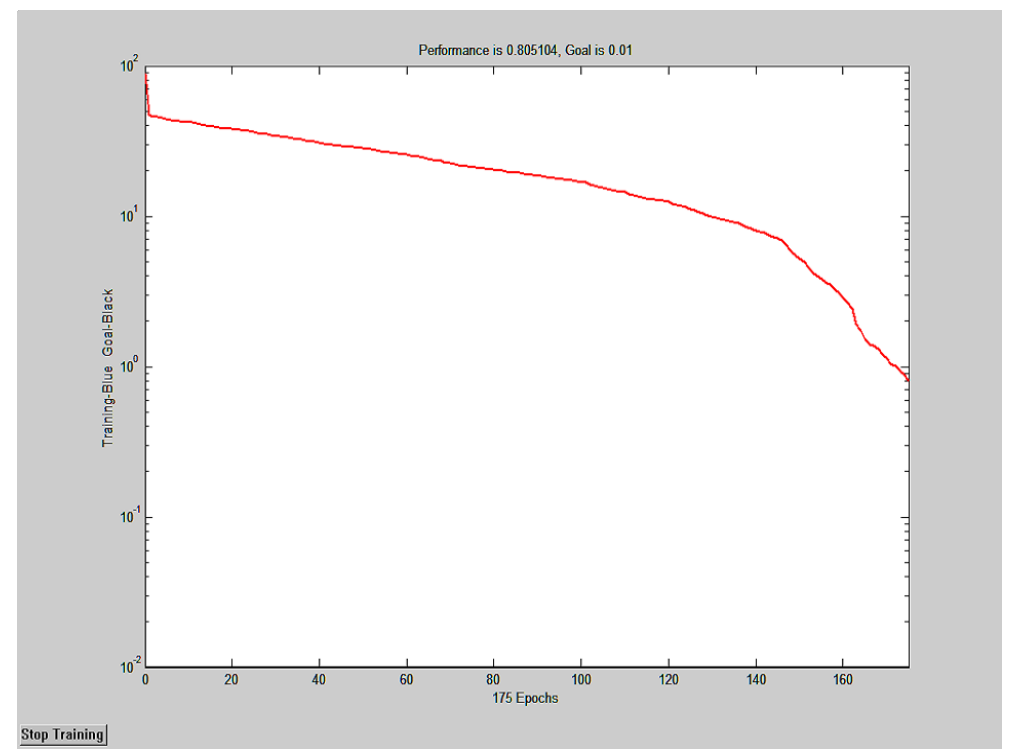

Figura 6: Entrenamiento de la red RBF

La precisión de la predicción, aumenta en la medida que la red neuronal es entrenada con un mayor número de datos. Por este motivo, es necesario ampliar las bases de datos disponibles con el fin de lograr una mejor capacidad de generalización del sistema. De los resultados obtenidos, se puede inferir que la permanencia de los alumnos en la institución, no depende solamente de las variables sicológicas demostradas mediante las pruebas de ingreso, sino que están implícitas variables exógenas que requieren ser modeladas.

El presente desarrollo, permite demostrar una vez más, la alta capacidad de generalización y clasificación de patrones que brindan los sistemas de inteligencia computacional al ser aplicados en la solución de problemas sico-sociales de alta complejidad, resaltando la eficiencia de las redes neuronales de base radial para resolver problemas de clasificación de patrones.

\section{Referencias}

[1] Freeman, J.A.; Skapura, D.M. (1991) Neural Networks. Algorithms, Aplications and Programming Techniques. Addison-Wesley, Reading.

[2] Masters, T. (1995) Advanced Algorithms for Neural Networks. John Wiley \& Sons, New York.

[3] Príncipe, J.; Euliano, N.; Lefebvre, C. (1999) Neural and Adaptive Systems. Jhon Wiley \& Sons, New York.

[4] Proakis, J.G., Manolakis, D.G. (1998) Tratamiento Digital de Señales, (3a Edición). Prentice Hall, Madrid. 


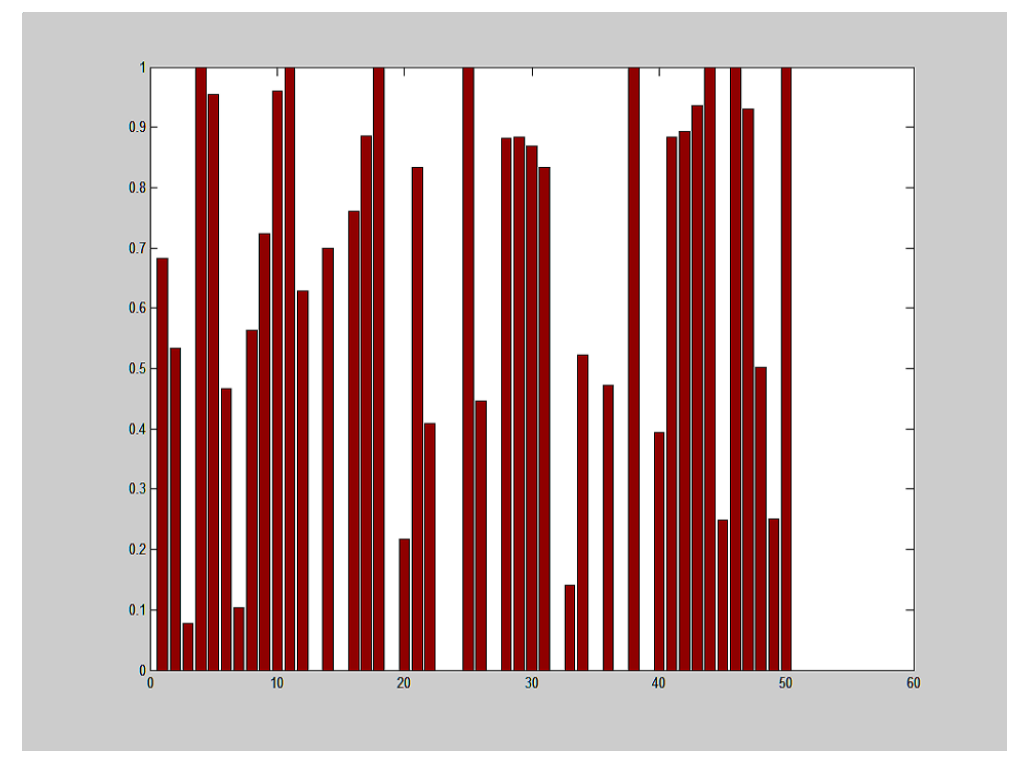

Figura 7: Grafica del error de validación

[5] Cattell, R.B. (1974) "How good is the modern questionnaire? General principles for evaluation", Journal of Personality Assessment 38(2): 115-129.

[6] Norgaard, M.; Ravn, O.; Poulsen, N.K.; Hansen, L.K. (2000) Neural Networks for Modelling and Control of Dynamic Systems. Springer-Verlag, London.

[7] García de Jalón ,J.; Rodríguez, J.I.; Brazález, A. (1999) Aprenda Matlab 5.3. Escuela Superior de Ingenieros Industriales, Universidad de Navarra.

[8] Oppenheim, A.V.; Burrus, C.S. (1998) Ejercicios de Tratamiento de la Señal Utilizando Matlab V. 4. Prentice Hall, Madrid.

[9] NATO Research and technology Organization (2001) "Final Report of the RTO Human Factors and Medicine Panel, (HFM) Research and Study Group 31 on Officer Selection. Printed by St. Joseph Ottawa/Hull, May. 